\title{
Photoluminescence Enhancement of Titanate Nanotubes by Insertion of Rare Earth Ions in Their Interlayer Spaces
}

\author{
Thalles M. F. Marques, ${ }^{1}$ Cleanio Luz-Lima, ${ }^{2}$ Marco Sacilloti, ${ }^{3}$ \\ Kazunori Fujisawa, ${ }^{4}$ Nestor Perea-Lopez, ${ }^{4}$ Mauricio Terrones, ${ }^{4}$ Eder Nascimento Silva, ${ }^{5}$ \\ Odair Pastor Ferreira, ${ }^{6}$ and Bartolomeu Cruz Viana ${ }^{1,2,4}$ \\ ${ }^{1}$ Pós-Graduação em Ciências dos Materiais, Universidade Federal do Piauí (UFPI), 64049-550 Teresina, PI, Brazil \\ ${ }^{2}$ Departamento de Física, Universidade Federal do Piauí (UFPI), 64049-550, Teresina, PI, Brazil \\ ${ }^{3}$ Departamento de Física, Universidade Federal de Pernambuco (UFPE), 50670-901 Recife, PE, Brazil \\ ${ }^{4}$ Department of Physics and Center for 2-Dimensional and Layered Materials, The Pennsylvania State University, \\ University Park, PA 16802, USA \\ ${ }^{5}$ Departamento de Física, CCET, Universidade Federal do Maranhão, 65085-580 São Luís, MA, Brazil \\ ${ }^{6}$ Laboratório de Materiais Funcionais Avançados (LaMFA), Departamento de Física, Universidade Federal do Ceará (UFC), \\ 60440-554 Fortaleza, CE, Brazil
}

Correspondence should be addressed to Odair Pastor Ferreira; opferreira@gmail.com and Bartolomeu Cruz Viana; bartolomeu@ufpi.edu.br

Received 27 July 2016; Revised 14 December 2016; Accepted 26 December 2016; Published 25 January 2017

Academic Editor: Giovanni Bongiovanni

Copyright (C) 2017 Thalles M. F. Marques et al. This is an open access article distributed under the Creative Commons Attribution License, which permits unrestricted use, distribution, and reproduction in any medium, provided the original work is properly cited.

\begin{abstract}
The optical properties of titanate nanotubes (TiNts) intercalated with rare earths (RE) ions were intensively investigated in this study. To prepare the nanomaterials, sodium titanate nanotubes (Na-TiNts) were submitted to ion exchange reactions with different rare earth elements (RE: $\mathrm{Pr}^{3+}, \mathrm{Er}^{3+}, \mathrm{Nd}^{3+}$, and $\mathrm{Yb}^{3+}$ ). To the best of our knowledge, it is the first time that these RE-TiNts were synthesized. All samples were characterized by Raman spectroscopy, X-ray powder diffraction (XRD), transmission electron microscopy (TEM), and energy dispersive X-ray spectroscopy (EDS). Furthermore, the optical properties were examined using photoluminescence spectroscopy (PL) and UV-Vis-NIR absorption spectroscopy. The PL intensity (visible range) of the RE-TiNt samples showed a strong dependence when the temperature was decreased down to $20 \mathrm{~K}$. This PL enhancement probably was promoted by electronic modifications in titanate layer band gap and/or interface charge transfers due to RE ions intercalation.
\end{abstract}

\section{Introduction}

Oxide based nanostructures exhibit some properties that differ from their bulk counterparts [1,2]. In particular, alkali metal titanates, usually possessing a layered or tunnel-type structure, have been subject of extensive investigations due to their excellent ion exchange ability added to the exceptional photocatalytic activities [3-6]. Kasuga et al. [7] demonstrated the possibility of producing alkali metal titanate nanoparticles with tubular morphology through hydrothermal treatment of $\mathrm{TiO}_{2}$ in the high alkaline medium. Ever since, titanate nanotubes (TiNts) have attracted both scientific and technological interest due to its unique combination of morphology, size, and functionality [8]. Furthermore, TiNts have large surface area $\left(\sim 180 \mathrm{~m}^{2} \cdot \mathrm{g}^{-1}\right)$ and porosity that leads to a wide variety of applications involving their surfaces [9$11]$.

Optical properties of nanomaterials are widely investigated due to their potential applications in small devices [1$3,8]$. The optical properties of TiNts have been tuned in according to the desired application using different methods to dope and/or inserting ions into the structure of the nanotubes $[3,6,7,12,13]$. For example, Li and Zhong [14] have 
inserted $\mathrm{Sn}^{2+}$ and $\mathrm{Pb}^{2+}$ in titanate nanotubes via ion exchange reaction and these nanomaterials were applied in photocatalysis using visible light for dyes degradation. Haro-González et al. [15] have prepared $\mathrm{Eu}^{3+}$ doped titania nanotubes via hydrothermal treatment of $\mathrm{Eu}^{3+}$ doped $\mathrm{TiO}_{2}$ nanopowders and they have studied the luminescent properties of these nanomaterials.

Photoelectronic properties of titanate nanotubes have been investigated [16]. It has been suggested that the photoluminescence emission process is from the exciton states trapped in titanate octahedral $\left(\left[\mathrm{TiO}_{6}\right]\right)$ units. The excitonic properties of titanate nanomaterials depend on the particular environment of the interlayer space. Therefore, the position and oxidation states of the cations intercalated can modify the $\left[\mathrm{TiO}_{6}\right]$ octahedron promoting or not excited states inside of its band gap with lifetime sufficient to have photoluminescence (PL) [16]. Then, there is a critical role of the layer composition and structure on charge separation of the photogenerated charge carriers. The intercalated ions can modify and control these properties of the layered nanostructures [16-18].

Rare earths (RE) doping and/or intercalating nanomaterials are widely investigated due to their excellent luminescent characteristics arising from the transitions of electronic levels which have optical applications such as lasers, optical amplifiers, phosphors, and other optical devices [19]. The choice of host materials has a great importance in the design of the rare earth-based luminescent properties for efficient applications $[8,20,21]$. In this work, in order to obtain different photoluminescence (PL) properties in titanate nanotubes, they were used as a host. $\mathrm{Na}^{+}$-intercalated titanate nanotubes (Na-TiNts) were prepared by hydrothermal method before being submitted to ion exchange reactions with different rare earth ions $\left(\mathrm{Pr}^{3+}, \mathrm{Er}^{3+}, \mathrm{Nd}^{3+}\right.$, and $\left.\mathrm{Yb}^{3+}\right)$. To the best of our knowledge, it is the first time that these nanotubes (Pr-, Er, Nd-, and Yb-TiNts) were prepared and their optical properties studied. Thus, this work proposes a very simple route to modify the optical properties of titanate nanotubes and obtain efficient PL emitters at room and low temperatures.

\section{Experimental Procedures}

All chemicals (reagent grade, Sigma-Aldrich, and Vetec) were used without further purification processes. Deionized water was used to prepare all solutions.

2.1. Titanate Nanotubes Preparation. Na-TiNts were prepared using commercial $\mathrm{TiO}_{2}$ (anatase) powder (purity of 99.8\%). Typically, $2.0 \mathrm{~g}$ of the $\mathrm{TiO}_{2}$ was mixed in $60 \mathrm{~mL}$ of $10 \mathrm{~mol} \mathrm{~L}^{-1}$ $\mathrm{NaOH}$ solution and then transferred into a Teflon-lined autoclave and heated at $150^{\circ} \mathrm{C}$ for $72 \mathrm{~h} \mathrm{[22].}$

2.2. Ion Exchange Reactions of the Titanate Nanotubes. The as-prepared $\mathrm{Na}$-TiNts are suitable for rare earths ion exchange reactions without additional process of protonation. These reactions were carried out by suspending $100 \mathrm{mg}$ of Na-TiNts in $100 \mathrm{~mL}$ of $0.05 \mathrm{~mol} \mathrm{~L}^{-1} \operatorname{Pr}\left(\mathrm{NO}_{3}\right)_{3} \cdot 6 \mathrm{H}_{2} \mathrm{O}$, $\mathrm{Nd}\left(\mathrm{NO}_{3}\right)_{3} \cdot 6 \mathrm{H}_{2} \mathrm{O}, \operatorname{Er}\left(\mathrm{NO}_{3}\right)_{3} \cdot 5 \mathrm{H}_{2} \mathrm{O}$, and $\mathrm{Yb}\left(\mathrm{NO}_{3}\right)_{3} \cdot 5 \mathrm{H}_{2} \mathrm{O}$ (purity of 99.9\%) aqueous solutions. After this, the suspension was left under magnetic stirring for $24 \mathrm{~h}$ at room temperature. The solid products were isolated by centrifugation at $3500 \mathrm{rpm}$. Then, the products were washed several times with deionized water aiming to remove residual soluble ions from the precursors. Finally, the samples were dried under vacuum conditions at room temperature during $6 \mathrm{~h}$ to obtain the RE intercalated titanate nanotubes (RE-TiNts).

2.3. Characterization. After the Na-TiNt and Re-TiNT preparations, the Raman spectroscopy experiments were performed using a Bruker Senterra spectrometer equipped with an Olympus BX5 microscope in all samples. A solid-state laser line at $532 \mathrm{~nm}$ was used for measuring the Raman spectra. Power density of laser was below $10^{7} \mathrm{~W} / \mathrm{m}^{2}$ to avoid sample overheating. A spectral resolution of $3 \mathrm{~cm}^{-1}$ was used and measurements were carried out using a backscattering geometry. X-ray powder diffraction (XRD) patterns were obtained using a Shimadzu XRD6000 diffractometer, using $\mathrm{CuK} \alpha(\lambda=1.5406 \AA)$ radiation operating at $30 \mathrm{~mA}$ and $40 \mathrm{kV}$. A scan rate of $1^{\circ} \mathrm{min}^{-1}$ was used. Transmission electron microscopy (TEM) images were obtained using a JEOL-JEM-2010F operating with $200 \mathrm{kV}$. The TEM samples were prepared by dropping aqueous suspension containing powder samples onto a holey carbon-coated copper grid and letting the water to evaporate at room temperature. The elemental analysis by energy dispersive X-ray spectroscopy (EDS) was performed using an Oxford X-ray detector attached to a Tescan FEG-Mira3 scanning electron microscope. The wavelength dependence of the optical diffuse reflectivity of the powder samples was measured using Perkin-Elmer Lambda 950 UV-Vis-NIR Spectrophotometer. Diffuse reflectance spectra were acquired using the Labsphere DRA-CA-30I accessory. Photoluminescence measurements were carried out upon excitation using an $\mathrm{Ar}^{+}$laser line at $488 \mathrm{~nm}$ on the powder pellet samples using a microscope with 50x objective lenses to focalize the laser beam. The emission signal was collected using the objective lenses and analyzed by a Renishaw inVia spectrometer equipped with an 1800 lines/mm grating and detected by a CCD. Lowtemperature measurements were performed in a helium flux cryostat (Advanced Research Systems). A digital temperature controller (Lakeshore) with $0.1 \mathrm{~K}$ of stability was used for monitoring the temperature in the range of $20-300 \mathrm{~K}$. The spectra were measured after an appropriate stabilization time for each thermal step.

\section{Results and Discussion}

In Figure 1(a) the Raman spectra of intercalated TiNts are shown. Firstly, the peaks around 158 and $190 \mathrm{~cm}^{-1}$ are assigned to lattice modes $\left(\mathrm{Na}^{+}-\mathrm{O}-\mathrm{Ti}\right)$. The peaks at 275,448 , 662 , and $700 \mathrm{~cm}^{-1}$ are assigned to Ti-O stretching in the edge-shared $\mathrm{TiO}_{6}$ and the peaks at $906 \mathrm{~cm}^{-1}$ represent the stretching vibration of the nonshared $\mathrm{Ti}-\mathrm{O}$ bonds in distorted $\mathrm{TiO}_{6}$ unit $[6,23,24]$. It was observed that the highest $\left(906 \mathrm{~cm}^{-1}\right)$ and the two lowest $\left(158\right.$ and $\left.190 \mathrm{~cm}^{-1}\right)$ energy 


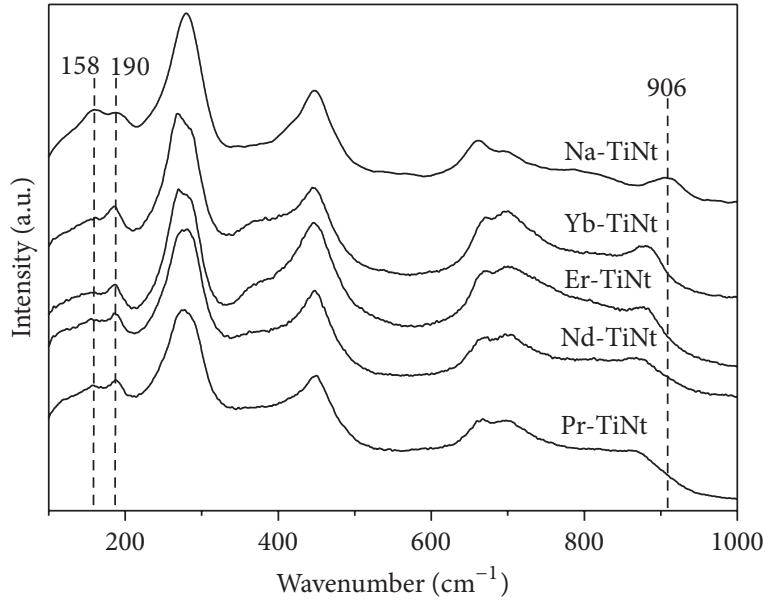

(a)

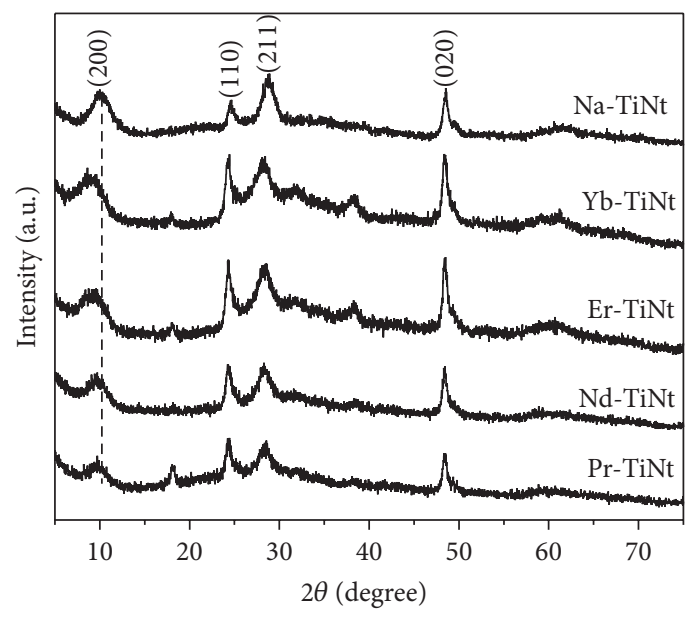

(b)

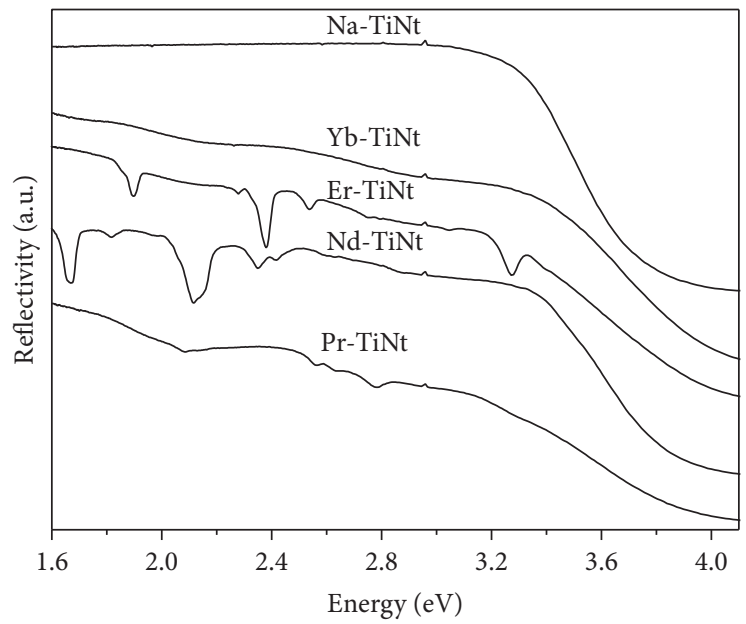

(c)

Figure 1: (a) Raman spectra; (b) X-ray diffractograms; and (c) UV-Vis reflectance spectra of Na-TiNts and RE-TiNts samples. The dashed lines on (a) and (b) parts are only eyes guide to show the displacement of the bands at 158, 190, and $906 \mathrm{~cm}^{-1}$ in Raman spectrum and (200) peak in diffractogram (peak related to interlayer spacing) of Na-TiNts after intercalation of RE ions, respectively.

modes have their wavenumbers and intensities extensively affected by the ion exchange with different RE ion species. Additionality, some of the medium energy modes (between 200 and $800 \mathrm{~cm}^{-1}$ ) are also changed. The most affected mode is that related to $\mathrm{Ti}-\mathrm{O}$ bonds whose oxygen atoms are not shared among the $\mathrm{TiO}_{6}$ units (at the corner of the $\mathrm{TiO}_{6}$ octahedral slab). Thus, the different RE ions inserted into the TiNts structure promote the reordering of the interlayer regions leading to these changes in Raman modes [25]. The changes can be explained as follows: the $\mathrm{Na}^{+}$ions were substituted by hydrated RE ions that may occupy different sites in the interlayer spaces. Due to this reordering the RE-O-Ti interlayer bond could deviate from a linear connection with the different intercalated ions and leads to shifting of the band at $906 \mathrm{~cm}^{-1}$ toward lower frequencies [25]. This explanation is supported by observing the difference between the electronegativity as well as the covalent radii of the $\mathrm{Na}$ and $\mathrm{RE}$ atoms. In general, the more electronegative ion will attract more easily the oxygen atoms, stretching the Ti-O bonds. Also, another important aspect to be pointed out is the considerable changes of the bands below $200 \mathrm{~cm}^{-1}$, which are related to the interlayer structure modifications. Thus, the coordination environments for $\mathrm{Na}^{+}$ion and other intercalated RE ions are different in the structural analysis, as also mentioned previously for other interlayer cations [23, 25].

To better understand the relationship between the crystalline structure and vibrational properties, $\mathrm{X}$-ray diffraction experiments were performed because they provide information about the interlayer region and crystallinity of the layers. The X-ray diffraction data (Figure 1(b)) suggested that both structure and morphology of TiNts were preserved after the $\mathrm{RE}$ ions intercalation. The peaks around $10^{\circ}(2 \theta)$ in $\mathrm{Na}-$ TiNts, attributed to (200) plane which is related to interlayer distance $[25,26]$, were slightly shifted to lower $2 \theta$ position when $\mathrm{RE}$ ions were intercalated indicating an increase of 
TABLE 1: RE/Ti atomic ratios obtained by EDS from intercalated samples. The amount of $\mathrm{Na}$ ions in pristine nanotubes is in agreement with the composition $\mathrm{Na}_{2-x} \mathrm{H}_{x} \mathrm{Ti}_{3} \mathrm{O}_{7} \cdot n \mathrm{H}_{2} \mathrm{O}$.

\begin{tabular}{lccccc}
\hline Samples & Na-TiNt & Pr-TiNt & Nd-TiNt & Er-TiNt & Yb-TiNt \\
\hline $\mathrm{Na} / \mathrm{Ti}$ & 0.33 & 0.02 & 0.01 & 0.01 & 0.01 \\
$\mathrm{RE} / \mathrm{Ti}$ & - & 0.19 & 0.14 & 0.18 & 0.17 \\
\hline
\end{tabular}

interlayer distance (see Table S1 in Supplementary Material available online at https://doi.org/10.1155/2017/3809807). This increase of the interlayer distance is expected since the ionic radius of $\mathrm{Na}^{+}$is smaller than that of $\mathrm{RE}$ hydrated ions [25, $27,28]$. Furthermore, it is possible to observe changes in the diagonal planes (110) and (211) which can be related to the rearrange of the RE ions into the layers of titanate to different coordination positions when it is compared with $\mathrm{Na}$-TiNt $[13,22]$.

Figure 1(c) shows the UV-Vis diffuse reflectance spectra for pristine nanotubes and RE-TiNts samples. It can be observed that Na-TiNts have only a strong band edge absorption in the ultraviolet region (above $3.0 \mathrm{eV}$ ). This absorption band in the ultraviolet range can be associated with the excitation of the O2p electron to the Ti3d level [29]. All of the RE-TiNts spectra have shown an edge band corresponding to the absorption edge of the titanate nanotubes at around $3.0 \mathrm{eV}$. However, for Pr-TiNts UV-Vis spectrum, it is possible to see three bands at $2.56,2.63$, and $2.78 \mathrm{eV}$ and a broad band at $2.08 \mathrm{eV}$ which indicates $\mathrm{Pr}^{3+}$ ions absorption peaks and that this ion was inserted into the TiNts [30]. The NdTiNts spectrum shows four broad and intense bands at 1.67, 2.11, 2.34, and $2.42 \mathrm{eV}$ attributed to $\mathrm{Nd}^{3+}$ ions absorptions [31]. In the spectrum of Er-TiNts bands can be seen at 1.89, $2.26,2.37,2.54$, and $3.28 \mathrm{eV}$ associated with trivalent erbium $\left(\mathrm{Er}^{3+}\right)$ absorption [32]. In the case of Yb-TiNts sample the absorption bands due to the ions absorption are not visible because it is out of the spectral range studied here; the absorption peak of $\mathrm{Yb}^{3+}$ ion is around $1.26 \mathrm{eV}[21,33]$. On the other hand, it is possible to see the $\mathrm{Yb}$-TiNts spectrum slightly broad in the edges around 2.25 and $2.95 \mathrm{eV}$ which could be associated with defects promoted in titanate electronic structure by ion exchanging [13]. The changes occurred in the TiNt spectrum profile when RE ions were inserted, like a slightly flat absorption on visible range, and a shifting of the absorption edge band can be attributed to charge transfer between TiNts host and RE ions, promoting modifications into the electronic structure of the titanate layers [34-36].

All analyses aforesaid indicate that pristine nanotubes after ion exchange reactions were modified due to substitution of Na by RE ions. To evaluate these substitutions directly, the chemical compositions of RE-TiNts samples were investigated by energy dispersive X-ray spectroscopy (EDS). $\mathrm{The} \mathrm{Na} / \mathrm{Ti}$ and $\mathrm{RE} / \mathrm{Ti}$ atomic ratios are shown in Table 1 . The amount of $\mathrm{Na}^{+}$ions in pristine nanotubes is in agreement with the $\mathrm{Na}_{2-x} \mathrm{H}_{x} \mathrm{Ti}_{3} \mathrm{O}_{7} \cdot n \mathrm{H}_{2} \mathrm{O}(0 \leq x \leq 2)$ chemical formula [22]. After ion exchange reactions, the sodium content drastically decreased in nanotubes. On the other hand, it was possible to detect the RE ions, suggesting that ion exchange reactions were very efficient to substitute $\mathrm{Na}$ ions by RE. These finding were demonstrated by a very low $\mathrm{Na} / \mathrm{Ti}$ atomic ratio in all $\mathrm{RE}$ ion exchanged samples and $\mathrm{RE} / \mathrm{Ti}$ atomic ratio between 0.14 and 0.19 . Also, the amount of oxygen and titanium in the samples was almost unchanged after the reactions. Then, $\left[(\mathrm{RE})_{2-x}\left(\mathrm{H}_{3 x}\right)\right]\left(\mathrm{Ti}_{3} \mathrm{O}_{7}\right)_{3} \cdot n \mathrm{H}_{2} \mathrm{O}$ chemical formula is suitable for RE-TiNts samples, disregarding the small amount of remaining $\mathrm{Na}^{+}$. Based on EDS analyses the following chemical formulas were proposed for RE-TiNts: $\operatorname{Pr}_{1.71} \mathrm{H}_{0.87}\left(\mathrm{Ti}_{3} \mathrm{O}_{7}\right)_{3} \cdot n \mathrm{H}_{2} \mathrm{O} ; \quad \mathrm{Nd}_{1.26} \mathrm{H}_{2.22}\left(\mathrm{Ti}_{3} \mathrm{O}_{7}\right)_{3} \cdot n \mathrm{H}_{2} \mathrm{O}$; $\mathrm{Er}_{1.62} \mathrm{H}_{1.14}\left(\mathrm{Ti}_{3} \mathrm{O}_{7}\right)_{3} \cdot n \mathrm{H}_{2} \mathrm{O}$; and $\mathrm{Yb}_{1.53} \mathrm{H}_{1.41}\left(\mathrm{Ti}_{3} \mathrm{O}_{7}\right)_{3} \cdot n \mathrm{H}_{2} \mathrm{O}$ $\left(\mathrm{H}^{+}\right.$was added in the chemical formulas because the aqueous solutions of rare earth salts are acids).

TEM images of the RE-TiNts (Figure 2) show that tubular morphology was preserved after the ion exchange reaction, corroborating with Raman and XRD results. Furthermore, in these images, it is not possible to observe nanoparticles decorating the nanotube on external surface as demonstrated in previous work about ion exchange reactions involving $\mathrm{Ce}^{4+}$ [6]. The RE-TiNts interlayer distances measured by TEM images $(0.72,0.83,0.86$, and $0.87 \mathrm{~nm}$ for Pr-, Er-, Nd-, and Yb-TiNts, resp.) are larger than $\mathrm{Na}$-TiNts one $(0.70 \mathrm{~nm}$, see Supplementary Material, Figure S1). Also, it is important to notice that, for all samples, the interlayer distances measured using TEM images are lower than those obtained by XRD patterns. These contractions would be due to partial release of water molecules present in the interlayer region [26,37], since for TEM experiments the samples were submitted to high vacuum conditions (below $10^{-6} \mathrm{~Pa}$ ).

In Figure 3(a) the photoluminescence spectra at room temperature and atmospheric pressure of the $\mathrm{Na}$-TiNts and RE-TiNts are shown. It is possible to see the PL enhancement of RE-TiNts as compared to pristine sample. Pr- and Er-TiNts had the highest luminescence intensity, being the emission band centered around 1.81 and $1.86 \mathrm{eV}$, respectively. Yb-TiNts had an emission around $2.04 \mathrm{eV}$ and the intensity is lower than Er-TiNts and Pr-TiNts. Finally, Nd-TiNts had the lowest PL intensity among the RE-TiNts samples with the broad emission band centered around $1.99 \mathrm{eV}$. Additionally, ErTiNts and Nd-TiNts spectra show that there are one $(1.89 \mathrm{eV})$ and three $(1.67,2.11$, and $2.14 \mathrm{eV})$ absorptions related to $\mathrm{Er}^{3+}$ [32] and $\mathrm{Nd}^{3+}$ [21].

The PL profiles are broad and typical of multiphonon process and/or interface emissions [35, 36, 38, 39]. PL spectra show a strong overlap of the peaks from the RE ions direct recombination as well, and the emission energies are lower than the direct recombination of an electron in Ti3d band with a hole in an $\mathrm{O} 2 \mathrm{p}$ band. Thus, electronic (defect) energy levels within the band gap and/or interface effects are promoted into TiNts by RE ions modifying the electronic structure of the host $[15,21]$. The electronic levels or interface densities of state were generated by the insertion of RE ions leading to the PL emission through of the TiNts lattice (host) (see Supplementary Information, Figure S2) [40].

Figure $3(\mathrm{~b})$ shows the effect of the vacuum $\left(\sim 10^{-5} \mathrm{~Pa}\right)$ on the PL spectra of Na-TiNts and RE-TiNts. It is possible 


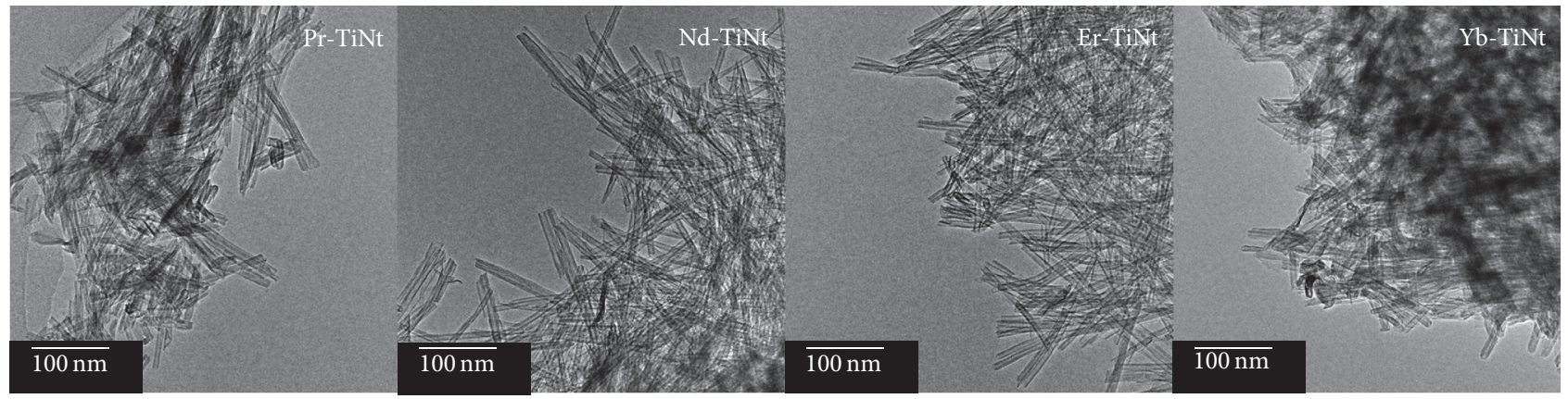

(a)

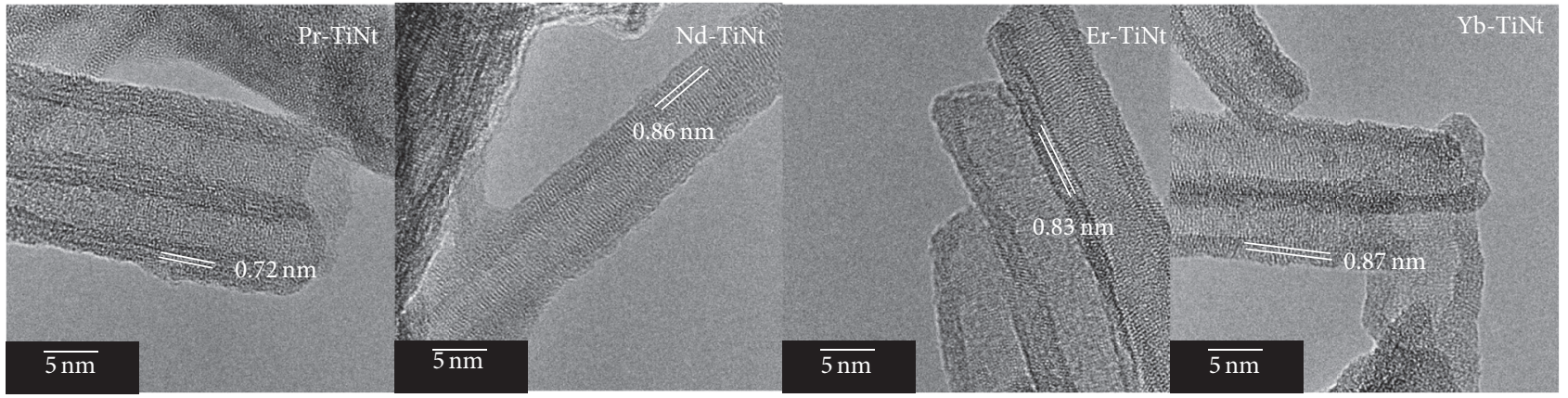

(b)

FIGURE 2: TEM (a) and HRTEM (b) images of RE-TiNts. The interlayer average distances of RE-TiNts samples were shown (white lines in HRTEM images).

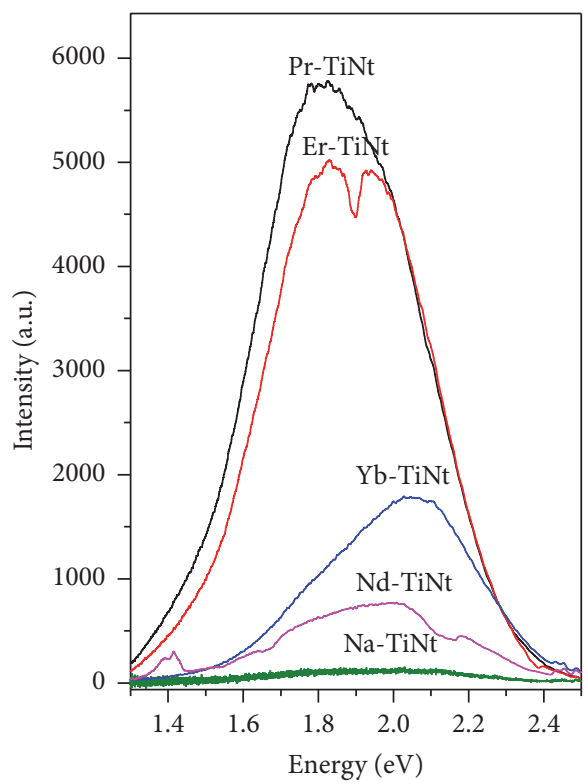

(a)

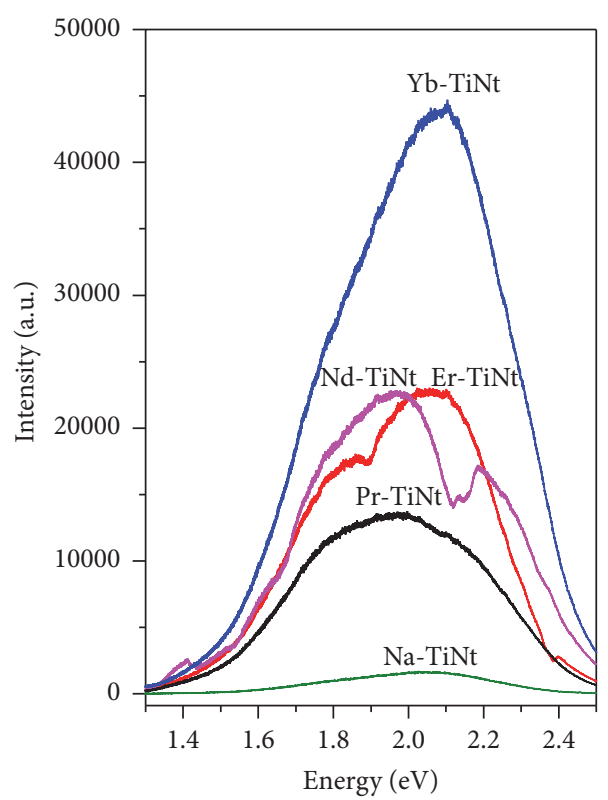

(b)

FIgure 3: Room temperature PL spectra of RE-TiNts and Na-TiNts samples at (a) atmospheric pressure and (b) under vacuum $\left(\sim 10^{-5} \mathrm{~Pa}\right)$. Some absorptions peaks due to RE ions inserted into the nanotubes can be seen. 
to see an enhancement of the PL intensity $(\sim 2, \sim 5, \sim 14$, $\sim 24$, and $\sim 30$ times more intense for $\mathrm{Pr}, \mathrm{Er}, \mathrm{Na}, \mathrm{Yb}$, and $\mathrm{Nd}$, resp.) probably, due to water release promoted by the vacuum conditions, suggesting that the interlayer space of the nanotubes was reordered and energy of ion-layer bonds and electronic levels were modified. Now, Yb-TiNts show the highest luminescence intensity with a more defined emission peak centered at $2.05 \mathrm{eV}$. For Er- and Pr-TiNts, emission peaks at $2.05 \mathrm{eV}$ and $1.95 \mathrm{eV}$ were observed, respectively. The spectrum of Nd-TiNts showed a peak around $1.95 \mathrm{eV}$. On the other hand, under vacuum Na-TiNts showed a weak broad PL peak around $2.05 \mathrm{eV}$. Some extra absorption peaks were observed on the spectra of $\mathrm{Yb}-$, $\mathrm{Nd}-$, and Er-TiNts, as it was observed in the PL spectra at atmospheric pressure. Thus, the water release and reordering of the interlayer space allowed another enhancement of the electronic recombination (PL) promoted by the excitation through inserted levels and/or interface densities of state into the electronic structure of TiNts intercalated with RE $[17,18,29]$.

Analyzing the PL of the RE-TiNts at atmospheric pressure (with structural water, Figure 3(a)), the intensity of PL peak could be related to the electronic effects promoted by the concentration of RE into the nanotube structures. The two most intense PL peaks are from Pr- and Er-TiNts, which have the highest concentration according to EDS quantifications (see Table 1). After the dehydration process, the electronic states promoted by vacancies compete with RE concentration changing the PL emission characteristics. Thus, it is very difficult to say, using that laser line $(488 \mathrm{~nm})$, which phenomena (electronic states inserted into the band gap of TiNts or interface energy states with charge transfer between RE and TiNts layers) can contribute the most to the PL emission efficiency. To understand better the PL mechanism, it is necessary to perform experiments with lower wavenumber laser lines to reach the energy of the band gap of pristine nanotubes.

The PL spectra at low temperatures were performed and they are shown in Figure 4. All of these PL peaks showed shifts with the decrease of temperature, and it may be due to some variations into the electronic structures. Furthermore, the intensity of PL peaks increase along with decreasing temperature which suggests that PL peaks could be related to shallow energy levels inserted by the ions and/or interface effects that are not thermally stable for electrons at that level [29]. In Na-TiNts spectrum (Figure 4(a)), the PL intensity increases almost linearly with the decrease of temperature and the peak position shifts to higher energies. The Nd-TiNts spectrum (Figure 4(b)) has shown the same behavior in the PL intensity and peak position, but there was stabilization at $45 \mathrm{~K}$ and the absorptions of $\mathrm{Nd}^{3+}$ ions are more evident at low temperatures. For Pr-TiNts (Figure 4(c)), during the decrease of temperature, the PL peak position shifts toward higher energies and PL intensity increases. Below $220 \mathrm{~K}$, an absorption peak appears around $2.08 \mathrm{eV}$ that can be related to $\mathrm{Pr}^{3+}$ direct absorption. For Er-TiNts (Figure 4(d)), the increase of intensity is almost linear as the temperature decreases and the peak position shows a shift to higher energy. Similar to NdTiNts, the $\mathrm{Er}^{3+}$ absorptions (at 1.89, 2.11, 2.26, and $2.34 \mathrm{eV}$ ) are more evident at low temperatures. Yb-TiNts spectrum (Figure 4(e)) has shown the same shift to higher energy at low temperatures. However, there was a decrease of the intensity from $35 \mathrm{~K}$ to $9 \mathrm{~K}$. This observation corroborates with an absorption plateau at UV-Vis measurements suggesting that $\mathrm{Yb}^{3+}$ ions modify the electronic structure of titanate nanotubes. As the emission process comes from electronic structure of the titanate nanotube walls which were affected by the RE insertion, this emission might be absorbed by the lanthanide ion into the interlayer promoting the absorption peaks in the PL spectra. The result of PL enhancement during the increase of temperature from $9 \mathrm{~K}$ to $35 \mathrm{~K}$ for $\mathrm{Yb}$-TiNts is probably due to thermal rectification of surface trap states or densities of states. A similar effect was observed for photoinduced PL efficiency enhancement of QDs during surface passivation $[35,41,42]$. At higher temperatures, the main part of nonradiative process that limits the quantum efficiency is the thermal escape, induced by multiple phonon absorption and promoting the PL quenching. At lower temperatures, the most of the excited electrons return to the shallow level via nonradiative transitions, which can lead to the PL enhancement [29].

\section{Conclusions}

In summary, we have reported the synthesis of titanate nanotubes intercalated with rare earths (RE) ions $\left(\mathrm{RE}: \mathrm{Pr}^{3+}, \mathrm{Er}^{3+}\right.$, $\mathrm{Nd}^{3+}$, and $\left.\mathrm{Yb}^{3+}\right)$. The morphology, structure, and optical properties of these novel titanate nanotubes were studied. The RE-TiNts samples have shown a higher PL emission than pristine Na-TiNts. The intensity and energy of the PL peaks are dependent on the temperature. The luminescence mechanism was briefly discussed and it was proposed that possible origin of the PL broad peaks would be due to the electronic levels inserted into the TiNts band gap and/or interface electronic densities introduced by the RE intercalations. Based on the results showed here, these novel nanomaterials could be used in applications involving optical devices like lasers, sensors, and phosphors.

\section{Competing Interests}

The authors declare that there is no conflict of interests regarding the publication of this paper.

\section{Acknowledgments}

The authors gratefully acknowledge financial support from the Brazilian funding agencies CNPq and FAPEPI and also acknowledge support from CAPES-PROCAD 2013 Grant 183995. 


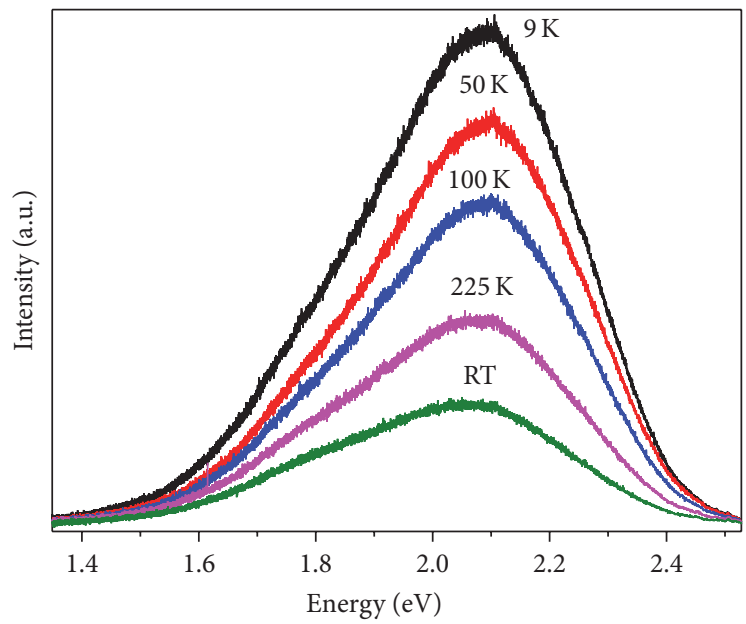

(a)

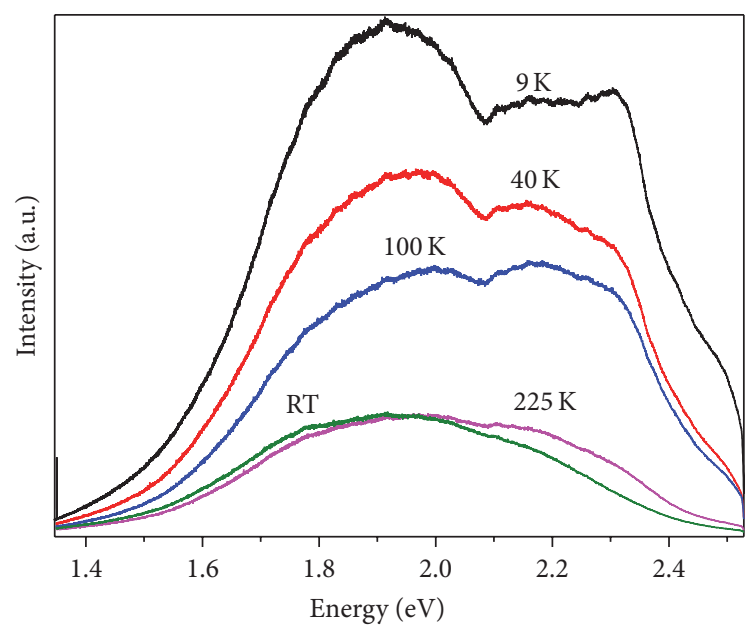

(c)

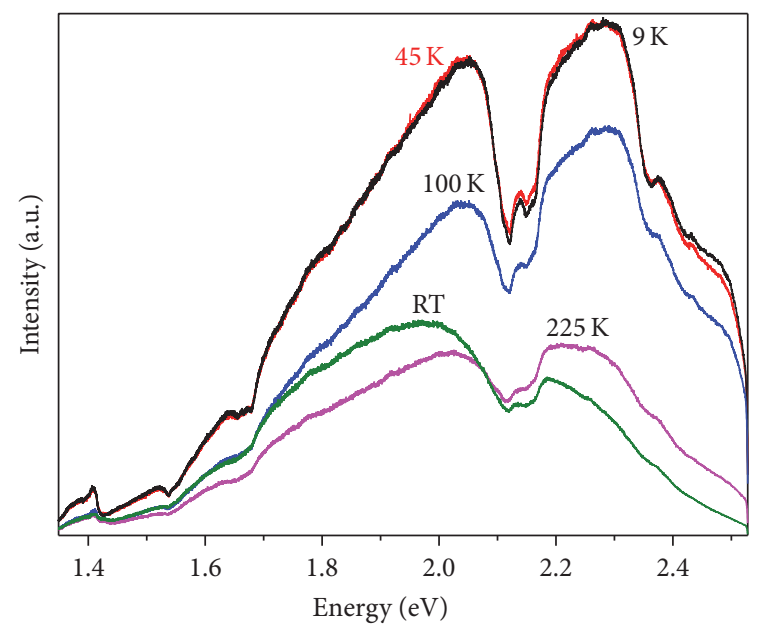

(b)

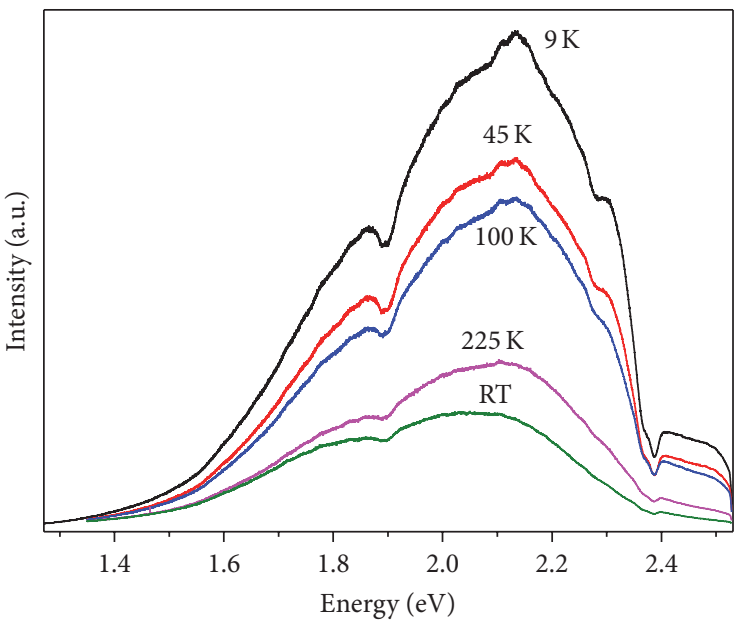

(d)

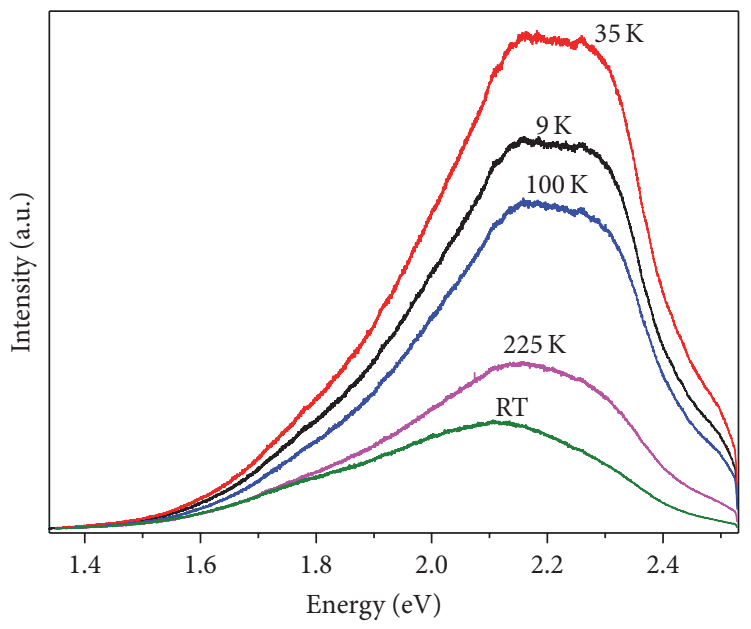

(e)

Figure 4: Temperature-dependent PL spectra: (a) Na-TiNts; (b) Nd-TiNts; (c) Pr-TiNts; (d) Er-TiNts; and (e) Yb-TiNts. 


\section{References}

[1] D. V. Bavykin and F. C. Walsh, "Elongated titanate nanostructures and their applications," European Journal of Inorganic Chemistry, vol. 2009, no. 8, pp. 977-997, 2009.

[2] A. Fujishima, K. Hashimoto, and T. Watanabe, $\mathrm{TiO}_{2}$ Photocatalysis: Fundamentals and Applications, BKC, 1999.

[3] X. Chen and S. S. Mao, "Titanium dioxide nanomaterials: Synthesis, properties, modifications and applications," Chemical Reviews, vol. 107, no. 7, pp. 2891-2959, 2007.

[4] V. Idakiev, Z.-Y. Yuan, T. Tabakova, and B.-L. Su, "Titanium oxide nanotubes as supports of nano-sized gold catalysts for low temperature water-gas shift reaction," Applied Catalysis A: General, vol. 281, no. 1-2, pp. 149-155, 2005.

[5] R. Tenne, "Inorganic nanotubes and fullerene-like nanoparticles," Nature Nanotechnology, vol. 1, no. 2, pp. 103-111, 2006.

[6] B. C. Viana, O. P. Ferreira, A. G. Souza Filho et al., "Decorating titanate nanotubes with $\mathrm{CeO} 2$ nanoparticles," Journal of Physical Chemistry C, vol. 113, no. 47, pp. 20234-20239, 2009.

[7] T. Kasuga, M. Hiramatsu, A. Hoson, T. Sekino, and K. Niihara, "Formation of titanium oxide nanotube," Langmuir, vol. 14, no. 12, pp. 3160-3163, 1998.

[8] M. Pedroni, F. Piccinelli, S. Polizzi, A. Speghini, M. Bettinelli, and P. Haro-González, "Upconverting $\mathrm{Ho}-\mathrm{Yb}$ doped titanate nanotubes," Materials Letters, vol. 80, pp. 81-83, 2012.

[9] N. M. dos Santos, J. M. Rocha, J. M. Matos et al., "Metal cations intercalated titanate nanotubes as catalysts for $\alpha, \beta$ unsaturated esters production," Applied Catalysis A: General, vol. 454, pp. 74-80, 2013.

[10] D. V. Bavykin, J. M. Friedrich, and F. C. Walsh, "Protonated titanates and $\mathrm{TiO}_{2}$ nanostructured materials: synthesis, properties, and applications," Advanced Materials, vol. 18, no. 21, pp. 2807-2824, 2006.

[11] R. Camposeco, S. Castillo, I. Mejia et al., "Active TiO2 nanotubes for CO oxidation at low temperature," Catalysis Communications, vol. 17, pp. 81-88, 2012.

[12] X. Ding, X. G. Xu, Q. Chen, and L.-M. Peng, "Preparation and characterization of Fe-incorporated titanate nanotubes," Nanotechnology, vol. 17, no. 21, pp. 5423-5427, 2006.

[13] X. Sun and Y. Li, "Synthesis and characterization of ionexchangeable titanate nanotubes," Chemistry-A European Journal, vol. 9, no. 10, pp. 2229-2238, 2003.

[14] X. Li and Y. Zhong, "Ion-exchangeable titanate nanotubes with visible light responsive photocatalytic activities for dyes degradation," Journal of Nanoscience and Nanotechnology, vol. 14, no. 9, pp. 7151-7156, 2014.

[15] P. Haro-González, M. Pedroni, F. Piccinelli et al., "Synthesis, characterization and optical spectroscopy of Eu3+ doped titanate nanotubes," Journal of Luminescence, vol. 131, no. 12, pp. 2473-2477, 2011.

[16] A. Riss, T. Berger, H. Grothe, J. Bernardi, O. Diwald, and E. Knözinger, "Chemical control of photoexcited states in titanate nanostructures," Nano Letters, vol. 7, no. 2, pp. 433-438, 2007.

[17] A. Riss, M. J. Elser, J. Bernardi, and O. Diwald, "Stability and photoelectronic properties of layered titanate nanostructures," Journal of the American Chemical Society, vol. 131, no. 17, pp. 6198-6206, 2009.

[18] A. Vittadini, M. Schirmer, M.-M. Walz et al., "Defects in oxygen-depleted titanate nanostructures," Langmuir, vol. 28, no. 20 , pp. 7851-7858, 2012.
[19] Z. Tang, L. Zhou, L. Yang, and F. Wang, "A study on the structure transformation and luminescence of Eu(III) titanate nanotubes synthesized at various hydrothermal temperatures," Journal of Alloys and Compounds, vol. 481, no. 1-2, pp. 704-709, 2009.

[20] B. Chi, E. S. Victorio, and T. Jin, "Synthesis of Eu-doped photoluminescent titania nanotubes via a two-step hydrothermal treatment," Nanotechnology, vol. 17, no. 9, pp. 2234-2241, 2006.

[21] X. Huang, S. Han, W. Huang, and X. Liu, "Enhancing solar cell efficiency: the search for luminescent materials as spectral converters," Chemical Society Reviews, vol. 42, no. 1, pp. 173-201, 2013.

[22] O. P. Ferreira, A. G. Souza, J. Mendes, and O. L. Alves, "Unveiling the structure and composition of titanium oxide nanotubes through ion exchange chemical reactions and thermal decomposition processes," Journal of the Brazilian Chemical Society, vol. 17, no. 2, pp. 393-402, 2006.

[23] H. Liu, D. Yang, E. R. Waclawik et al., "A Raman spectroscopic study on the active site of sodium cations in thestructure of $\mathrm{Na}_{2} \mathrm{Ti}_{3} \mathrm{O}_{7}$ during the adsorption ofSr ${ }^{2+}$ and $\mathrm{Ba}^{2+}$ cations," Journal of Raman Spectroscopy, vol. 41, no. 12, pp. 1792-1796, 2010.

[24] Y. Su, M. L. Balmer, and B. C. Bunker, "Raman spectroscopic studies of silicotitanates," Journal of Physical Chemistry B, vol. 104, no. 34, pp. 8160-8169, 2000.

[25] B. C. Viana, O. P. Ferreira, A. G. Filho, A. A. Hidalgo, J. M. Filho, and O. L. Alves, "Alkali metal intercalated titanate nanotubes: a vibrational spectroscopy study," Vibrational Spectroscopy, vol. 55, no. 2, pp. 183-187, 2011.

[26] Q. Chen, G. H. Du, S. Zhang, and L.-M. Peng, "The structure of trititanate nanotubes," Acta Crystallographica, Section B: Structural Science, vol. 58, no. 4, pp. 587-593, 2002.

[27] R. D. Shannon and C. T. Prewitt, "Effective ionic radii in oxides and fluorides," Acta Crystallographica Section B, vol. 25, no. 5, pp. 925-946, 1969.

[28] Y. Matsumoto, U. Unal, Y. Kimura, S. Ohashi, and K. Izawa, "Synthesis and photoluminescent properties of titanate layered oxides intercalated with lanthanide cations by electrostatic selfassembly methods," Journal of Physical Chemistry B, vol. 109, no. 26, pp. 12748-12754, 2005.

[29] S. Luo, J. Fan, W. Liu et al., "Synthesis and low-temperature photoluminescence properties of $\mathrm{SnO}_{2}$ nanowires and nanobelts," Nanotechnology, vol. 17, no. 6, pp. 1695-1699, 2006.

[30] X. Li, B. Yang, J. Zhang, L. Hu, and L. Zhang, "Energy transfer between Er3+ and Pr3+ for $2.7 \mu \mathrm{m}$ fiber laser material," Fibers, vol. 2, no. 1, pp. 24-33, 2014.

[31] A. I. Gavrilov, N. A. Belich, S. V. Shuvaev, D. O. Gil', B. R. Churagulov, and E. A. Gudilin, "Synthesis of perovskite sodium neodymium titanates and study of their photocatalytic properties," Doklady Chemistry, vol. 454, no. 1, pp. 9-12, 2014.

[32] M. A. Meneses-Nava, O. Barbosa-García, J. L. Maldonado et al., " $\mathrm{Yb}^{3+}$ quenching effects in co-doped polycrystalline $\mathrm{BaTiO}_{3}: \mathrm{Er}^{3+}, \mathrm{Yb}^{3+}$," Optical Materials, vol. 31, no. 2, pp. 252-260, 2008.

[33] M. K. Mahata, K. Kumar, and V. K. Rai, "Structural and optical properties of Er3+/Yb3+ doped barium titanate phosphor prepared by co-precipitation method," Spectrochimica Acta A: Molecular and Biomolecular Spectroscopy, vol. 124, pp. 285-291, 2014.

[34] S. Okamoto and H. Yamamoto, "Luminescent properties of praseodymium-doped alkaline-earth titanates," Journal of Luminescence, vol. 102-103, pp. 586-589, 2003. 
[35] H. Zhu, N. Song, and T. Lian, "Wave function engineering for ultrafast charge separation and slow charge recombination in type II core/shell quantum dots," Journal of the American Chemical Society, vol. 133, no. 22, pp. 8762-8771, 2011.

[36] P. Dorenbos, "Systematic behaviour in trivalent lanthanide charge transfer energies," Journal of Physics Condensed Matter, vol. 15, no. 49, pp. 8417-8434, 2003.

[37] Y. Suzuki and S. Yoshikawa, "Synthesis and thermal analyses of $\mathrm{TiO}_{2}$-derived nanotubes prepared by the hydrothermal method," Journal of Materials Research, vol. 19, no. 4, pp. $982-$ 985, 2004.

[38] M. Sacilotti, D. Chaumont, C. B. Mota et al., "Interface recombination \& emission applied to explain photosynthetic mechanisms for $(\mathrm{e}-, \mathrm{h}+)$ Charges' separation," World Journal of Nano Science and Engineering, vol. 2, pp. 58-87, 2012.

[39] S. Kumar, M. Jones, S. S. Lo, and G. D. Scholes, "Nanorod heterostructures showing photoinduced charge separation," Small, vol. 3, no. 9, pp. 1633-1639, 2007.

[40] V. M. Longo, M. das Graça Sampaio Costa, A. Zirpole Simões et al., "On the photoluminescence behavior of samarium-doped strontium titanate nanostructures under UV light. A structural and electronic understanding," Physical Chemistry Chemical Physics, vol. 12, no. 27, pp. 7566-7579, 2010.

[41] L. An, J. Yi, C. Liu, W. An, and Y. Zhang, "Room and Low temperature photoluminescence properties of CdSe quantum dots synthesized rapidly in aqueous approach," in Proceedings of the International Conference on Electronics and Optoelectronics (ICEOE '11), pp. 195-198, Liaoning, China, July 2011.

[42] G. Morello, M. De Giorgi, S. Kudera, L. Manna, R. Cingolani, and M. Anni, "Temperature and size dependence of nonradiative relaxation and exciton-phonon coupling in colloidal CdTe quantum dots," Journal of Physical Chemistry C, vol. 111, no. 16, pp. 5846-5849, 2007. 

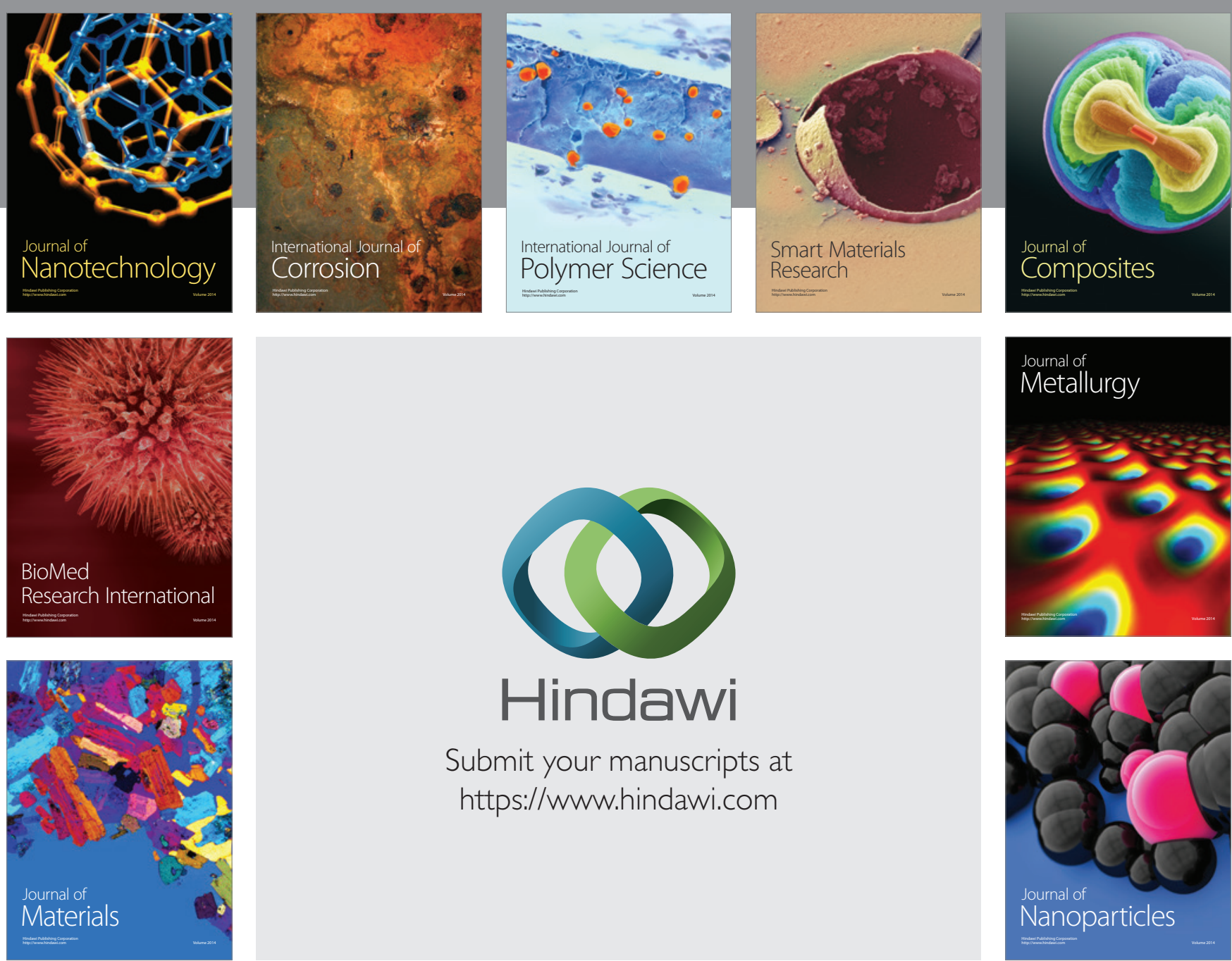

\section{Hindawi}

Submit your manuscripts at

https://www.hindawi.com

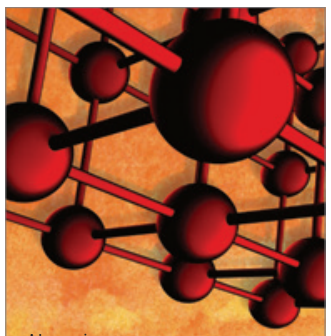

Materials Science and Engineering
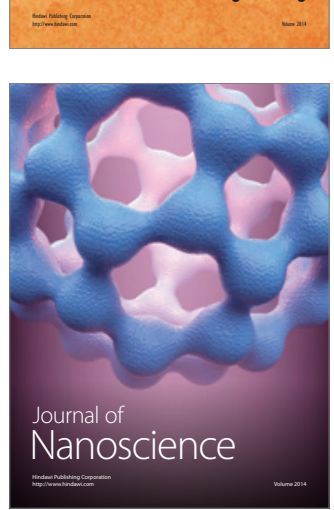
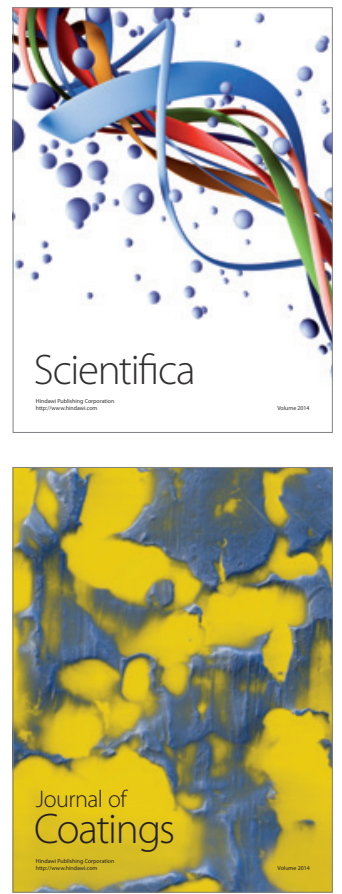
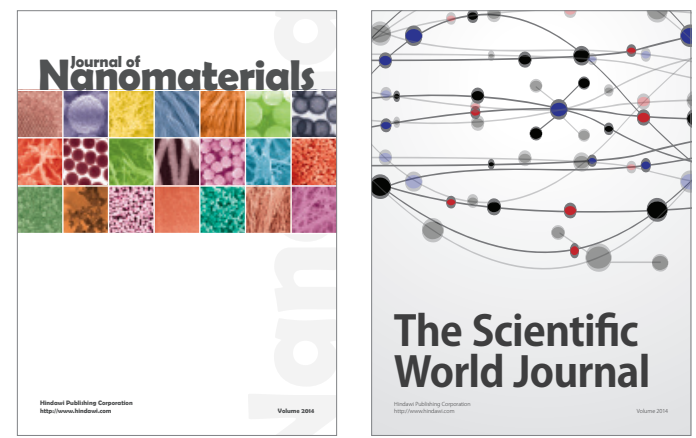

The Scientific World Journal
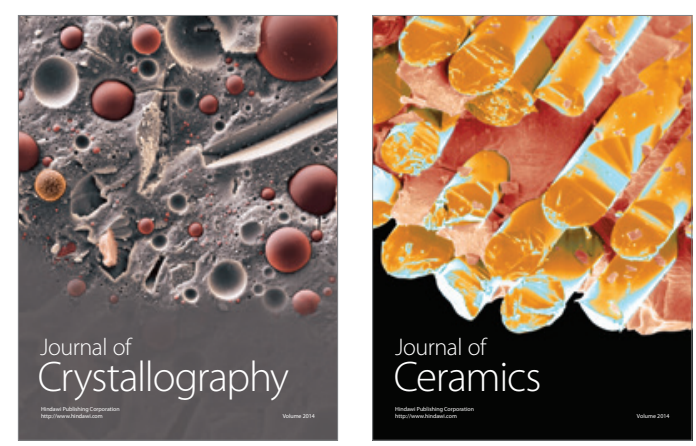
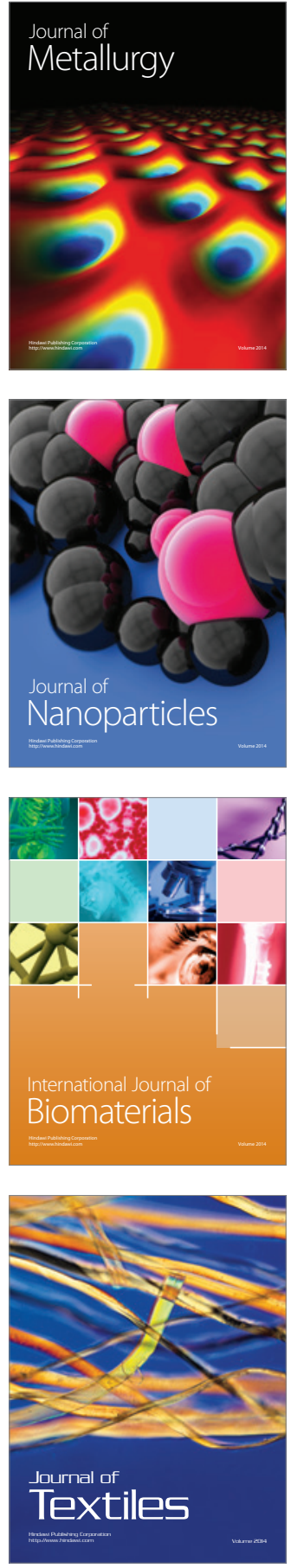